\title{
Nonlinear Control of Tendon Driven Robot Manipulators: Elimination of Actuator Side Position Measurements
}

\author{
Beytullah Okur, Erkan Zergeroglu and Enver Tatlicioglu
}

\begin{abstract}
In this study, a partial state feedback controller is proposed for the link position tracking control problem of flexible tendon driven robotic systems. Specifically; a nonlinear model based controller is formulated for tendon driven robot manipulators under the constraint that only the link position and tendon expansion force measurements are available. Despite the lack of link and actuator side velocity and actuator position measurements, the proposed controller ensures exponential link position tracking. To eliminate the need of actuator position and velocity measurements, a model based velocity observer has been utilized. Stability of the closed loop system and boundedness of system states are proven via Lyapunov based arguments. The performance of the purposed observer-controller couple is then verified by a set of numerical simulations.
\end{abstract}

\section{INTRODUCTION}

Tendon transmission systems present clean, shock absorbent and have less noisy characteristics compared to other power transmission systems. Owing to these advantages tendon driven mechanisms can be used in many applications to separate the actuators from the robot links as actuating each joint remotely would decrease the link size, mass and inertia. Previous research on this field have been conducted on both small size and large scale robotic systems. To name some; [1],[2],[3] can be given as an example to small size applications like robotic hands and [4], [5], [6] are some examples for large size manipulators. However, in applications where the main performance criteria is to track a desired trajectory in the task space, the use of tendon driven mechanisms are limited. This is mostly due to the elastic nature of the tendons and the extra dynamical terms inserted to the system due to this nonlinear elasticity. For accurate position control performance, considering the whole system dynamics in the controller design is essential. For tendon driven systems, it necessary to include the elastic tendon dynamics to the system model nevertheless with this inclusion control problem becomes more complicated due to the extra dynamics [5] [7].

For some background on tendon driven robot manipulators and classical linear control approaches on tendon driven systems, the reader is referred to [8], [9],[10] and references

This research is supported by Grants of the Scientific and Technological Research Council of Turkey, TUBITAK Project No: 112E561.

B. Okur is with Department of Electronics Engineering,Gebze Institute of Technology, 41400, Gebze, Kocaeli, Turkey. beytullahokuregyte.edu.tr

E. Zergeroglu is with the Department of Computer Engineering, Gebze Institute of Technology, 41400, Gebze, Kocaeli, Turkey. ezergerebilmuh.gyte.edu.tr

E. Tatlicioglu is with the Department of Electrical \& Electronics Engineering, Izmir Institute of Technology, 35430, Urla, Izmir, Turkey enverdiyte.edu.tr therein. Focusing on the model based controllers for tendon driven systems, formulations including system dynamics are limited. In [5] Kobayashi and Ozawa presented adaptive and an adaptive neural-network based controller for tendon driven robotic mechanisms with elastic tendons. In [11], Nakayama and Fujimoto tackled the tracking control of tendon driven robots by applying the delayed reflexive force feedback. In [12] and [13], Haiya et al. proposed controller for multiple degree-of-freedom tendon mechanisms using nonlinear springs with hysteresis characteristics. For the proposed controllers, error of the equation of spring was estimated by a disturbance observer and compensated by utilizing the estimated disturbance. In recent years, backstepping type model based controllers including tendon elasticity in the system model have been published [7], [14], [15].

In this study, a nonlinear model based partial state feedback controller for tendon driven robot manipulators that does not require neither acceleration nor velocity measurements. More over purposed controller do not need actuator side position measurements. The purposed controller only requires link position measurements, tensile force measurements of each tendons and exact knowledge of the model parameters. Need for link velocity measurements are eliminated by utilizing a nonlinear link velocity filter during the error system development and the lack of actuator position and velocity measurements have been overcome with the help of a model based velocity filter design. Taking into consideration of the tendon elasticity and power transmission dynamics results a more complicated overall system model which requires usage of the backstepping technique twice. The semi-global exponential stability of the link position error and boundedness of all the signals under the closed loop operation have been proved using Lyapunov type analysis tools and performance of the purposed controller is verified via numerical simulations.

Compared to our previous studies, the proposed controller does not require the full state information as [7]. And when compared to [14] the proposed controller removes the need of actuator side position measurements. However while the adaptive controller of [14] can coupe for the system uncertainties, the proposed controller requires the exact knowledge of the system dynamics.

The rest of the paper is organised as follows. The modelling of the tendon driven robot manipulator is given in Section II. In Section III error system development and controller-observer formulations are presented in detail. Stability analysis is given Section IV. Section V presents the simulation results and finally our conclusions can be seen in 
Section VI.

\section{System DyNAMICS AND PROPERTIES}

The dynamics of $n-$ DOF robot manipulator driven by an $m$-tendon mechanism is assumed to have the following form [5]

$$
\begin{gathered}
M(q) \ddot{q}+V_{m}(q, \dot{q}) \dot{q}+F_{d} \dot{q}+G(q)=-J_{j}^{T}(q) f_{t}(l) \\
J \ddot{\theta}+B \dot{\theta}+R_{a} f_{t}(l)=\tau_{a} \\
\dot{l}=J_{j}(q) \dot{q}+R_{a} \dot{\theta}
\end{gathered}
$$

where $q(t), \dot{q}(t), \ddot{q}(t) \in R^{n}$ represent the link position, velocity and acceleration vectors respectively, $\theta(t), \dot{\theta}(t), \ddot{\theta}(t) \in$ $R^{m}$ represents the actuator position, velocity and acceleration vectors respectively, $l, i \in R^{m}$ are the $m$-dimensional tendon expansion vector and its time derivative, $M(q) \in$ $R^{n \times n}$ denotes the link inertia effects, $V_{m}(q, \dot{q}) \in R^{n \times n}$ represents the centripedal-Coriolis effects, $G(q) \in R^{n}$ denotes the gravitational terms related to the robot, $F_{d} \in R^{n \times n}$ is the constant diagonal link viscous friction matrix, $J, B \in R^{m \times m}$ are the diagonal actuator inertia and actuator viscous friction matrices respectively, $R_{a} \in R^{m \times m}$ is the diagonal matrix containing the radius of pulleys mounted on each actuator, $J_{j}(q) \in R^{m \times n}$ Jacobian matrix that maps the joint space to the tendon expansion space, $f_{t}(l) \in R^{m}$ is the vector of tendon tensile forces generated by the tendon expansions and finally $\tau_{a} \in R^{m}$ is the control input vector applied the actuators. The dynamic equations of (1), exhibit the following useful properties, which will be utilized in the controller development and the subsequent stability analysis;

Property 1: The inertia matrix can be bounded from above and below by the following inequalities [16]

$$
m_{1} I_{n} \leq M(q) \leq m_{2} I_{n}
$$

where $m_{1}$ and $m_{2}$ are positive constants, and $I_{n}$ is the $n \times n$ identity matrix. Likewise the inverse of of the inertia matrix can be bounded as follows

$$
\frac{1}{m_{2}} I_{n} \leq M^{-1}(q) \leq \frac{1}{m_{1}} I_{n}
$$

Property 2: The inertia and the centripetal-Coriolis matrices satisfy the following relationship [17]

$$
\xi^{T}\left(\frac{1}{2} \dot{M}(q)-V_{m}(q, \dot{q})\right) \xi=0 \quad \forall \xi \in R^{n}
$$

where $\dot{M}(q)$ represents the time derivative of the inertia matrix.

Property 3: The centripetal-Coriolis matrix satisfies the following relationship [18]

$$
V_{m}(q, v) \xi=V_{m}(q, \xi) v \quad \forall \xi, v \in R^{n} .
$$

Property 4: The norm of the centripetal-Coriolis and friction matrices can be upper bounded as follows [16]

$$
\begin{aligned}
\left\|V_{m}(q, \xi)\right\|_{i \infty} & \leq \zeta_{c 1}\|\xi\|, \quad\left\|F_{d}\right\|_{i \infty} \leq \zeta_{f}, \\
\|G(q)\| & \leq \zeta_{g} \quad \forall \xi \in R^{n} .
\end{aligned}
$$

where $\zeta_{c 1}, \zeta_{f}$, and $\zeta_{g}$ are known positive bounding constants and $\|\cdot\|_{i \infty}$ denotes the infinity norm of a matrix.
Property 5: The robot dynamics given in (1) can be linearly parameterized as follows [16]

$$
Y(q, \dot{q}, \ddot{q}) \phi_{r}=M(q) \ddot{q}+V_{m}(q, \dot{q}) \dot{q}+G(q)+F_{d} \dot{q}
$$

where $\phi_{r} \in R^{p}$ contains the constant system parameters, and $Y(q, \dot{q}, \ddot{q}) \in R^{n \times p}$ denotes the regression matrix that is a function only of $q(t), \dot{q}(t)$, and $\ddot{q}(t)$. The formulation of (10) can also written in terms of the desired trajectory in the following manner

$$
\begin{aligned}
Y_{d}\left(q_{d}, \dot{q}_{d}, \ddot{q}_{d}\right) \phi_{r}= & M\left(q_{d}\right) \ddot{q}_{d}+V_{m}\left(q_{d}, \dot{q}_{d}\right) \dot{q}_{d} \\
& +G\left(q_{d}\right)+F_{d} \dot{q}_{d}
\end{aligned}
$$

where the desired regression matrix $Y_{d}\left(q_{d}, \dot{q}_{d}, \ddot{q}_{d}\right) \in R^{n \times p}$ is a function of the desired link position, velocity, and acceleration vectors denoted by $q_{d}(t), \dot{q}_{d}(t), \ddot{q}_{d}(t) \in R^{n}$, respectively.

\section{Problem Statement and Controller FORMULATION}

The control objective is to design a link position tracking controller for the tendon driven robot manipulator model given by (1),(2) and (3) under the constraints that acceleration and velocity measurements of links and actuators and more over position measurements of actuators are not available. Specifically, the controller should force the robot links to exponentially follow a desired trajectory despite the lack of link/actuator velocity measurements and actuator position measurements. In order to quantify the control objective, we define the link position tracking error $e(t) \in \mathbb{R}^{n}$ as follows

$$
e \triangleq q_{d}-q
$$

where it is assumed that the desired link position signal, $q_{d}(t)$ and its first four time derivatives are sufficiently smooth and bounded functions of time. We define a filtered tracking error-like term $\eta(t) \in \mathbb{R}^{n}$ as follows

$$
\eta=\dot{e}+\alpha_{1} e+\alpha_{2} e_{f}
$$

where $\alpha_{1}, \alpha_{2}$ are positive constant filter gains and $e_{f}(t) \in \mathbb{R}^{n}$ is an auxiliary filter variable having the following dynamic relationship

$$
\dot{e}_{f}=-\alpha_{3} e_{f}+\alpha_{2} e-k \eta ; \quad e_{f}(0)=0
$$

where $\alpha_{3}$ is a positive constant filter gain, $k$ is apositive constant control gain. After taking the time derivative of (13), premultiplying both sides by $M(q)$, substituiting (1), (13) and (14), adding and substructing $Y_{d} \phi_{r}$ and $V_{m}(q, \dot{q}) \eta$ to the right side of the resulting equation, we obtain the following expression.

$$
\begin{aligned}
M(q) \dot{\eta}= & -V_{m}(q, \dot{q}) \eta+Y_{d} \phi_{r}-\alpha_{2} k M(q) \eta \\
& +J_{j}^{T}(q) f_{t}(l)+\chi
\end{aligned}
$$


where

$$
\begin{aligned}
\chi= & M(q) \ddot{q}_{d}+V_{m}\left(q, \dot{q}_{d}\right) \dot{q}_{d}+F_{d} \dot{q}+G(q) \\
& -Y_{d} \phi_{r}+\alpha_{1} M(q)\left(\eta-\alpha_{1} e-\alpha_{2} e_{f}\right) \\
& +\alpha_{2} M(q)\left(-\alpha_{3} e_{f}+\alpha_{2} e\right) \\
& -V_{m}(q, \eta)\left(\dot{q}_{d}+\alpha_{1} e+\alpha_{2} e_{f}\right) \\
& +V_{m}\left(q, \dot{q}_{d}\right)\left(\alpha_{1} e+\alpha_{2} e_{f}\right) \\
& +V_{m}\left(q, \dot{q}_{d}+\alpha_{1} e+\alpha_{2} e_{f}\right)\left(\alpha_{1} e+\alpha_{2} e_{f}\right) .
\end{aligned}
$$

Based on the bounds of the model matrices, boundedness of the desired trajectory and its derivatives and several properties of the robot dynamics it can be shown that

$$
\|\chi\| \leq \rho_{0}\|x\|+\rho_{1}\|x\|^{2}
$$

where $\rho_{0}, \rho_{1}$ are positive bounding constants, and $x(t) \in \mathbb{R}^{3 n}$ is defined as follows

$$
x=\left[\begin{array}{lll}
e^{T} & e_{f}^{T} & \eta^{T}
\end{array}\right]^{T} .
$$

In order to apply backstepping procedure to reach the tendon dynamics we define an auxiliary error term $\eta_{f}(t) \in \mathbb{R}^{m}$ as follows

$$
\eta_{f}=f_{t}(l)-f_{d}
$$

where $f_{d}(t) \in \mathbb{R}^{m}$ is an auxiliary control input. Adding and substructing $J_{j}^{T}(q) f_{d}$ to the equation (15) we have the following form.

$$
\begin{aligned}
M(q) \dot{\eta}= & -V_{m}(q, \dot{q}) \eta+Y_{d} \phi_{r}-\alpha_{2} k M(q) \eta \\
& +\chi+J_{j}^{T}(q) \eta_{f}+J_{j}^{T}(q) f_{d} .
\end{aligned}
$$

To stabilize the open loop robot dynamics given in (20) we design the axillary control input $f_{d}$ according to the subsequent stability analysis as follows.

$$
f_{d} \triangleq\left[J_{j}^{T}(q)\right]^{+}\left\{-Y_{d} \phi_{r}+k K_{s} e_{f}-K_{s} e\right\}
$$

where $(.)^{+}$is used to represent the pseudo inverse of a matrix $K_{s}$ is a positive constant diagonal control gain matrix and the control gain $k$ is defined as follows

$$
k=\frac{1}{m_{1}}\left[1+k_{n 1}\left(\rho_{0}^{2}+\rho_{1}^{2}\right)\right]
$$

where $k_{n 1}$ is a positive constant nonlinear damping gain and $m_{1}$ was defined in (4). Closed loop dynamics can be obtained as follows.

$$
\begin{aligned}
M(q) \dot{\eta}= & -V_{m}(q, \dot{q}) \eta-\alpha_{2} k M(q) \eta \\
& +\chi+J_{j}^{T}(q) \eta_{f}+k K_{s} e_{f}-K_{s} e .
\end{aligned}
$$

From (23) and subsequent stability analysis, it can be seen that to ensure the stability and the convergence of the tracking error signal we need to ensure $\eta_{f}(t)$ converges to zero.
A. Model based observer design for actuator side velocity measurements

From the actuator dynamics given in (2) we obtain the following expression

$$
\ddot{\theta}=J^{-1}\left[\tau_{a}-B \dot{\theta}-R_{a} f_{t}(l)\right] .
$$

To write the actuator dynamics in a useful state space form we define state variables $x_{1}(t)=\theta, x_{2}(t)=\dot{\theta} \in \mathbb{R}^{m}$ and obtain the state space form of actuator dynamics as follows

$$
\left[\begin{array}{c}
\dot{x}_{1} \\
\dot{x}_{2}
\end{array}\right]=\left[\begin{array}{c}
x_{2} \\
J^{-1} \tau_{a}-J^{-1} B x_{2}-J^{-1} R_{a} f_{t}(l)
\end{array}\right] .
$$

According to the subsequent stability analysis we design the actuator side velocity observer as follows

$$
\begin{gathered}
\dot{\hat{x}}_{2}=J^{-1} \tau_{a}-J^{-1} B \hat{x}_{2}-J^{-1} R_{a} f_{t}(l) \\
+\frac{\partial f_{t}(l)}{\partial l} R_{a} \eta_{f}+\Omega_{4} \eta_{b}
\end{gathered}
$$

Observation error can be obtained in the following manner

$$
\dot{\tilde{x}}_{2}=\dot{x}_{2}-\dot{\hat{x}}_{2}=-J^{-1} B \tilde{x}_{2}-\frac{\partial f_{t}(l)}{\partial l} R_{a} \eta_{f}-\Omega_{4} \eta_{b} .
$$

From the closed loop dynamics of $\eta$ given by (23) and observation error dynamics given by (27) it is clear that we need to show that auxiliary error signal $\eta_{f}$ converges to zero. Taking the time derivative of (19),

$$
\begin{aligned}
\dot{\eta}_{f}= & \frac{\partial f_{t}(l)}{\partial l}\left[J_{j}(q) \dot{q}+R_{a} \dot{\theta}\right] \\
& -\left[J_{j}^{T}(q)\right]^{+}\left\{-\dot{Y}_{d} \phi+k K_{s} \dot{e}_{f}-K_{s} \dot{e}\right\} \\
& -\frac{d}{d t}\left[J_{j}^{T}(q)\right]^{+}\left\{-Y_{d} \phi+k K_{s} e_{f}-K_{s} e\right\} .
\end{aligned}
$$

The time derivative of the pseudo inverse of the Jacobian matrix which can be seen in last line of the previous equation can be formulated by the sum of two functions: one of them includes only time dependent terms and the other includes terms of the multiplier of $\eta$. So the last line of the previous equation can be separated as follows

$$
\frac{d}{d t}\left[J_{j}^{T}(q)\right]^{+}\{\cdot\} \triangleq \Psi_{1}\left(e, e_{f}, t\right)+\Psi_{2}\left(e, e_{f}, t\right) \eta .
$$

It is clear that if the Jacobian matrix is not a function of time this part will be zero. To investigate the dynamics of $\eta_{f}$, we make use of equations (13), (14), (29) and add and subtruct $\frac{\partial f_{t}(l)}{\partial l} R_{a} \hat{x}_{2}$ to obtain

$$
\dot{\eta}_{f}=\Omega_{0}+\Omega_{1} \eta+\frac{\partial f_{t}(l)}{\partial l} R_{a} \tilde{x}_{2}+\frac{\partial f_{t}(l)}{\partial l} R_{a} \hat{x}_{2}
$$

where

$$
\begin{aligned}
\Omega_{0}= & \frac{\partial f_{t}(l)}{\partial l} J_{j}(q)\left(\dot{q}_{d}+\alpha_{1} e+\alpha_{2} e_{f}\right)-\Psi_{1} \\
& -\left[J_{j}^{T}(q)\right]^{+}\left[-\dot{Y}_{d} \phi+k K_{s}\left(-\alpha_{3} e_{f}+\alpha_{2} e\right)\right] \\
& +\left[J_{j}^{T}(q)\right]^{+} K_{s}\left(-\alpha_{1} e-\alpha_{2} e_{f}\right)
\end{aligned}
$$

and

$$
\Omega_{1}=-\frac{\partial f_{t}(l)}{\partial l} J_{j}(q)+\left[J_{j}^{T}(q)\right]^{+}\left(k K_{s} k+K_{s}\right)-\Psi_{2} .
$$


Here $\Omega_{0} \in \mathbb{R}^{m}$ and $\Omega_{1} \in \mathbb{R}^{m \times n}$ include known and measurable terms. We define a new auxiliary input tracking error $\eta_{b}(t) \in$ $\mathbb{R}^{m}$ as follows

$$
\eta_{b}(t) \triangleq \hat{x}_{2}-x_{2 d}
$$

And we apply back-stepping procedure to the open loop dynamics of $\eta_{f}$ by adding and subtracting $\frac{\partial f_{t}(l)}{\partial l} R_{a} x_{2 d}$

$$
\begin{aligned}
\dot{\eta}_{f}= & \Omega_{0}+\Omega_{1} \eta+\frac{\partial f_{t}(l)}{\partial l} R_{a} \tilde{x}_{2} \\
& +\frac{\partial f_{t}(l)}{\partial l} R_{a} \eta_{b}+\frac{\partial f_{t}(l)}{\partial l} R_{a} x_{2 d}
\end{aligned}
$$

According to the subsequent stability analysis we design auxiliary input $u_{L}$ in the following manner

$$
x_{2 d}=\Lambda\left(-K_{f} \eta_{f}-\Omega_{0}\right)
$$

where the auxiliary variable $\Lambda(l) \in \mathbb{R}^{m \times m}$ is defined as

$$
\Lambda \triangleq\left[\frac{\partial f_{t}(l)}{\partial l} R_{a}\right]^{-1}
$$

and $K_{f} \in \mathbb{R}^{m \times m}$ is the controller gain matrix and defined as

$$
K_{f} \triangleq k_{f}+k_{n 2}\left\|\Omega_{1}\right\|_{i \infty}^{2}+k_{n 3} \lambda_{\max }\left(J_{j}^{T}(q)\right)^{2}
$$

where $k_{f} \in \mathbb{R}^{m \times m}$ is a constant, diagonal control gain matrix, $k_{n 2}, k_{n 3} \in \mathbb{R}^{1}$ are constant scalar nonlinear damping gains. After substituting (35) in to the (34) closed loop dynamics for $\eta_{f}$ can be obtained as

$$
\dot{\eta}_{f}=-K_{f} \eta_{f}+\Omega_{1} \eta+\frac{\partial f_{t}(l)}{\partial l} R_{a} \tilde{x}_{2}+\frac{\partial f_{t}(l)}{\partial l} R_{a} \eta_{b}
$$

In this step we continue the control design to show that auxiliary error signal $\eta_{b}$ converges to zero. To investigate the dynamics of $\eta_{b}$ we take the time derivative of (33), take the other necessary derivatives in the resulting equation and than substitute (26) (30), (3), (13) and do some more mathematical manipulations to obtain

$$
\dot{\eta}_{b}=\Omega_{2}+\Omega_{3} \eta+\Omega_{4} x_{2}+J^{-1} \tau_{a}
$$

where $\Omega_{2} \in \mathbb{R}^{m}, \Omega_{4} \in \mathbb{R}^{m \times m}$ and $\Omega_{3} \in \mathbb{R}^{m \times n}$ include the known terms. According to the subsequent stability analysis we design the actuator control input as follows

$$
\tau_{a}=J\left\{-K_{b} \eta_{b}-\Omega_{2}-\Omega_{4} \hat{x}_{2}-\frac{\partial f_{t}(l)}{\partial l} R_{a} \eta_{f}\right\}
$$

with

$$
K_{b}=k_{b}+k_{n 4}\left\|\Omega_{3}\right\|_{i \infty}^{2}
$$

where $k_{b} \in \mathbb{R}^{m \times m}$ is a constant, diagonal control gain matrix, $k_{n 4} \in \mathbb{R}^{1}$ is constant scalar nonlinear damping gain. After substituting (40) in to the (39) closed loop dynamics for $\eta_{b}$ can be obtained as

$$
\dot{\eta}_{b}=-K_{b} \eta_{b}+\Omega_{3} \eta+\Omega_{4} \tilde{x}_{2}-\frac{\partial f_{t}(l)}{\partial l} R_{a} \eta_{f}
$$

\section{Stability AnAlysis}

The stability of the closed loop system will be investigated by utilizing Lyapunov-based arguments. Semi-global exponential stability of the link position tracking error is presented in the following theorem.

Theorem 1: For the tendon driven robot manipulator system given by dynamic equations (1), (2) and (3), link position tracking adaptive controllers given by (21), (35), (40) used in conjunction with link velocity error generating filter given by (13), (14) and actuator velocity observer given by (26) ensures the semi-global exponential stability of the link position tracking error in the sense that

$$
\|e(t)\| \leq \sqrt{\frac{\lambda_{2}}{\lambda_{1}}\|z(0)\|^{2} \exp (-\beta t)}
$$

where $z(t) \in R^{3 n+3 m}$ is defined as follows

$$
z \triangleq\left[\begin{array}{cccc}
x^{T} & \tilde{x}_{2}^{T} & \eta_{f}^{T} & \eta_{b}^{T}
\end{array}\right]^{T}
$$

and provided the nonlinear damping gains are selected to satisfy the following conditions

$$
k_{n}>\frac{\lambda_{2}}{\lambda_{1}}\|z(0)\|^{2}+1
$$

where $\lambda_{1} \in \mathbb{R}^{1}, \lambda_{2} \in \mathbb{R}^{1}$ are defined as follows

$$
\begin{aligned}
& \lambda_{1} \triangleq \frac{1}{2} \min \left\{m_{1}, \lambda_{\min }\left(K_{s}\right), 1\right\} \\
& \lambda_{2} \triangleq \frac{1}{2} \max \left\{m_{2}, \lambda_{\max }\left(K_{s}\right), 1\right\}
\end{aligned}
$$

Note that $\lambda_{\min }(\cdot)$ and $\lambda_{\max }(\cdot)$ are used to denote the minimum eigenvalue and maximum eigenvalue of a matrix.

Proof: We define a non-negative Lyapunov-like function $V(t)$ as follows

$$
\begin{aligned}
V= & \frac{1}{2} \eta^{T} M(q) \eta+\frac{1}{2} e^{T} K_{s} e+\frac{1}{2} e_{f}^{T} K_{s} e_{f} \\
& +\frac{1}{2} \eta_{f}^{T} \eta_{f}+\frac{1}{2} \eta_{b}^{T} \eta_{b}+\frac{1}{2} \tilde{x}_{2}^{T} \tilde{x}_{2}
\end{aligned}
$$

which can be bounded from below and above as follows

$$
\lambda_{1}\|z\|^{2} \leq V \leq \lambda_{2}\|z\|^{2} .
$$

Taking the time derivative of (48) along (13), (14), (23), (38) and (42) then using the well known skew symmetric property, cancelling the same terms, using the definition of gains $k, K_{f}$ and $K_{b}$ given in (22), (37) and (41), applying upper bouds with the help of the nonlinear damping tool, we can write the following upper bound for $\dot{V}$

$$
\begin{aligned}
\dot{V} \leq & -\alpha_{2}\|\eta\|^{2}-\alpha_{1} K_{s}\|e\|^{2}-\alpha_{3} K_{s}\left\|e_{f}\right\|^{2} \\
& -k_{f}\left\|\eta_{f}\right\|^{2}-k_{b}\left\|\eta_{b}\right\|^{2}-J^{-1} B\left\|\tilde{x}_{2}\right\|^{2} \\
& +\frac{1}{4 \alpha_{2} k_{n 1}}\|x\|^{2}+\frac{1}{4 \alpha_{2} k_{n 1}}\|x\|^{4} \\
& +\frac{\|\eta\|^{2}}{4 k_{n 2}}+\frac{\left\|\tilde{x}_{2}\right\|^{2}}{4 k_{n 3}}+\frac{\|\eta\|^{2}}{4 k_{n 4}} .
\end{aligned}
$$


Using the definition of $z$ given in (44), upper bound can be simplified as follows

$$
\dot{V} \leq-\left(\beta-\frac{1}{k_{n}}\left(1+\|z\|^{2}\right)\right)\|z\|^{2}
$$

where $\beta, k_{n}$ are some positive constants. Making use of bounds of $V$ given in (50) results the final form for the upper bound for $\dot{V}$ as

$$
\dot{V} \leq-\left(\beta-\frac{1}{k_{n}}\left(1+\frac{V}{\lambda_{1}}\right)\right)\|z\|^{2} .
$$

To conclude the negative semi-definiteness of $\dot{V}$, the term in the outer parenthesis need to be positive. That is we must have

$$
V \leq \lambda_{1}\left(k_{n}-1\right)
$$

for (53) to be negative semi-definite. This requirement ensures the negative semi-definiteness of (53) but makes our stability region semi-global [19]. From (53) and (54) the resulting upper bound for $\dot{V}$ can be obtained as

$$
\dot{V} \leq-\gamma\|z\|^{2}
$$

where $\gamma$ is a some positive bounding constant. The expression given in (55) can be further upper bounded by the help of using the definition of $z(t)$ and the upper bound of $V(t)$ given in (50) as follows

$$
\dot{V} \leq-\delta V
$$

where $\delta$ is a some positive bounding constant. The solution of the above differential inequality yields

$$
V(t) \leq V(0) \exp (-\delta t)
$$

and from direct application of (50) we can obtain the following upper bound for $z(t)$

$$
\|z(t)\| \leq \sqrt{\frac{\lambda_{2}}{\lambda_{1}}\|z(0)\|^{2} \exp (-\delta t)}
$$

where $\lambda_{1}, \lambda_{2}$ were previously defined in (46) and (47). Based on the definition of $z(t)$ and (58), it can be shown that the tracking error term $e(t)$ is bounded as stated in (43). Moreover applying standard signal chasing argument we can show that all signals in the closed-loop error system are bounded.

\section{Simulations}

To see the performance of the purposed controller we make simulations on a two link robotic arm which is driven by 6 tendons as shown in Fig. (1). The 2 link robotic manipulator has the following model matrices according to the (1)

$$
\begin{gathered}
M=\left[\begin{array}{cc}
p_{1}+2 p_{3} \cos \left(q_{2}\right) & p_{2}+p_{3} \cos \left(q_{2}\right) \\
p_{2}+p_{3} \cos \left(q_{2}\right) & p_{2}
\end{array}\right] \\
V_{m}=\left[\begin{array}{cc}
-p_{3} \sin \left(q_{2}\right) \dot{q}_{2} & -p_{3} \sin \left(q_{2}\right)\left(\dot{q}_{1}+\dot{q}_{2}\right) \\
p_{3} \sin \left(q_{2}\right) \dot{q}_{1} & 0
\end{array}\right] \\
F_{d}=\left[\begin{array}{cc}
f_{d 1} & 0 \\
0 & f_{d 2}
\end{array}\right]
\end{gathered}
$$

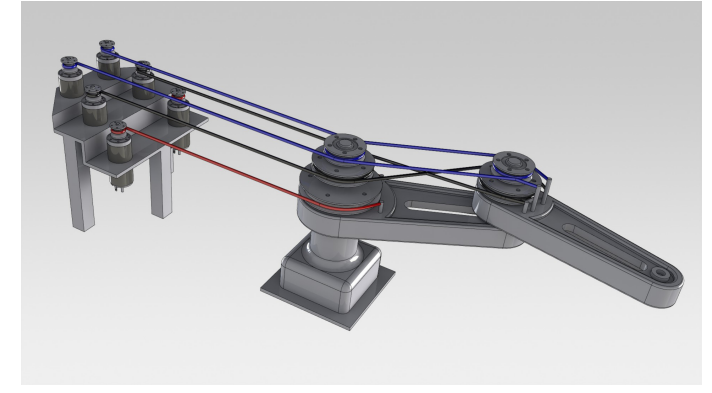

Fig. 1. 2 link planar robot driven by 6 tendons
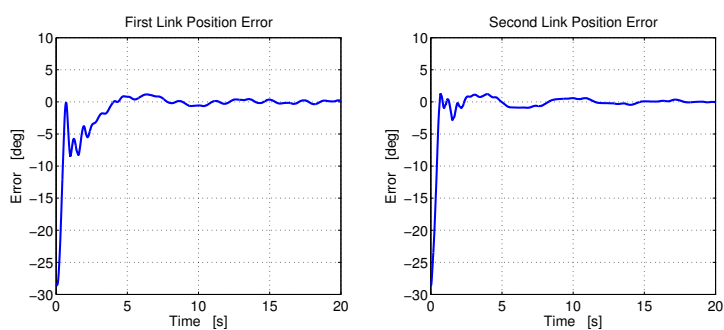

Fig. 2. Link Errors

and the Jocobian matrix is defined as follows [5]

$$
J_{j}^{T}(q)=s_{1}\left[\begin{array}{cccccc}
1 & -1 & 1 & -1 & 1 & -1 \\
0 & 0 & -1 & 1 & 1 & -1
\end{array}\right] .
$$

Tendon tensile forces can be calculated as a function of tendon expansions in the following manner [5]

$$
f_{t}\left(l_{i}\right)=\left\{\begin{array}{cc}
s_{2} l_{i}+s_{3} l_{i}^{3} & l_{i} \geqslant 0 \\
0 & l_{i}<0
\end{array}\right\}, i=1, . ., 6
$$

where $l_{i}$ are the tendon expansions and $f_{t}\left(l_{i}\right)$ are the members of the vector of the tendon tensile forces which is defined as follows

$$
f_{t}(l)=\left[\begin{array}{llllll}
f_{t}\left(l_{1}\right) & f_{t}\left(l_{2}\right) & f_{t}\left(l_{3}\right) & f_{t}\left(l_{4}\right) & f_{t}\left(l_{5}\right) & f_{t}\left(l_{6}\right)
\end{array}\right]^{T}
$$

The robot parameters are taken as $p_{1}=0.006 \mathrm{kgm}^{2}, p_{2}=$ $0.003 \mathrm{kgm}^{2}, p_{3}=0.002 \mathrm{kgm}^{2}, f_{d 1}=0.005 \mathrm{Nm} * \mathrm{sec}, f_{d 2}=$ $0.001 \mathrm{Nm} * \mathrm{sec}, s_{1}=0.015, s_{2}=7907.5 \mathrm{~N} / \mathrm{m}$ and $s_{3}=$ $1.7898 \times 10^{8} \mathrm{~N} / \mathrm{m}^{3}$ [5] for the simulations. The actuator part of the tendon mechanism has identical six de motors which have the inertia of $0.142 \mathrm{kgm}^{2}$ and viscous friction of $0.025 \mathrm{Nm} * \mathrm{sec} / \mathrm{rad}$ and the pulleys mounted on the actuators have radius of $10 \mathrm{~cm}$. The desired trajectory of the robot is selected as $q_{d 1}=q_{d 2}=0.5 \sin (t)\left(1-\exp \left(-3 t^{3}\right)\right) \mathrm{rad}$ and initial positions of the robot links are set to $0.5 \mathrm{rad}$. Controller gain matrices are selected as $\alpha_{1}=\operatorname{diag}\{6,6\}$, $\alpha_{2}=\operatorname{diag}\{1.5,1.5\}, \alpha_{3}=\operatorname{diag}\{1,1\}, k=\operatorname{diag}\{0.9,0.8\}$, $K_{s}=\operatorname{diag}\{13,15\}, K_{f}=100 I_{6}$ and $K_{b}=100 I_{6}$. The simulation results are shown in Figures 2-4. Figure 2 shows the link position tracking errors. Figure 3 presents the control torques applied to each actuator, while Figure 4 presents the tendon tensile forces.

\section{CONCLUSIONS}

In this study, a nonlinear model based partial state feedback controller for a flexible tendon driven robotic manipu- 

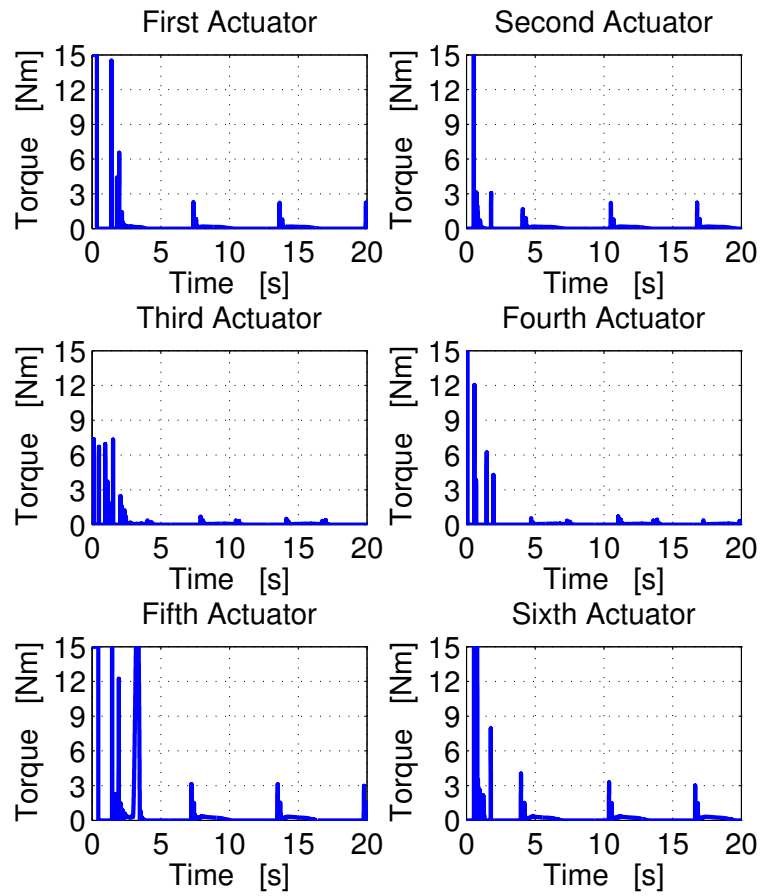

Fig. 3. Actuator inputs

lator with the exact knowledge of the model parameters have been presented. Despite the unavailability of the velocity measurements of both links and actuators and position measurements of the actuators, the proposed controller ensured that the link trajectory tracking error signal converges to zero exponentially. Semi-global stability of the closed-loop system and boundedness of system states are proven via Lyapunov based arguments. Simulation studies are performed to illustrate the effectiveness and viability of the proposed method.

\section{REFERENCES}

[1] E. K. I. S. C. Jacobsen, H. Ko and C. C. Davis, "Antagonistic control of a tendon driven manipulator," in Proc. of IEEE Int. Conf. on Robotics and Automation, 1989, pp. 1334-1339.

[2] W. P. M. Kaneko and H. Tolle, "Input-dependent stability of joint torque control of tendon-driven robot hands," IEEE Tr. on Industrial Electronics, vol. 39, no. 2, pp. 96-104, 1992.

[3] M. T. Mason and J. K. S. Jr., Robot Hands and the Mechanics of Manipulation. Cambridge, MA, USA: MIT Press, 1985.

[4] S. H. S. Ma and H. Yashinada, "Design and experiments for a coupled tendon-driven manipulator," IEEE Control Systems Magazine, vol. 13, no. 1 , pp. 30-36, 1993.

[5] H. Kobayashi and R. Ozawa, "Adaptive neural network control of tendon driven mechanisms with elastic tendons," Automatica, vol. 39, pp. 1509-1519, 2003.

[6] J. L. S. Chang and H. Yen, "Kinematic and compliance analysis for tendon driven robotic mechanisms with flexible tendons," Mechanism and Machine Theory, vol. 40, pp. 728-739, 2005.

[7] B. Okur, O. Aksoy, E. Zergeroglu, and E. Tatlicioglu, "Nonlinear robust control of tendondriven robot manipulators," Journal of Intelligent and Robotic Systems, pp. 1-12, 2014. [Online]. Available: http://dx.doi.org/10.1007/s10846-014-0141-7

[8] R. Ozawa, H. Kobayashi, and K. Hashirii, "Analysis, classification, and design of tendon-driven mechanisms," Robotics, IEEE Transactions on, vol. 30, no. 2, pp. 396-410, April 2014.
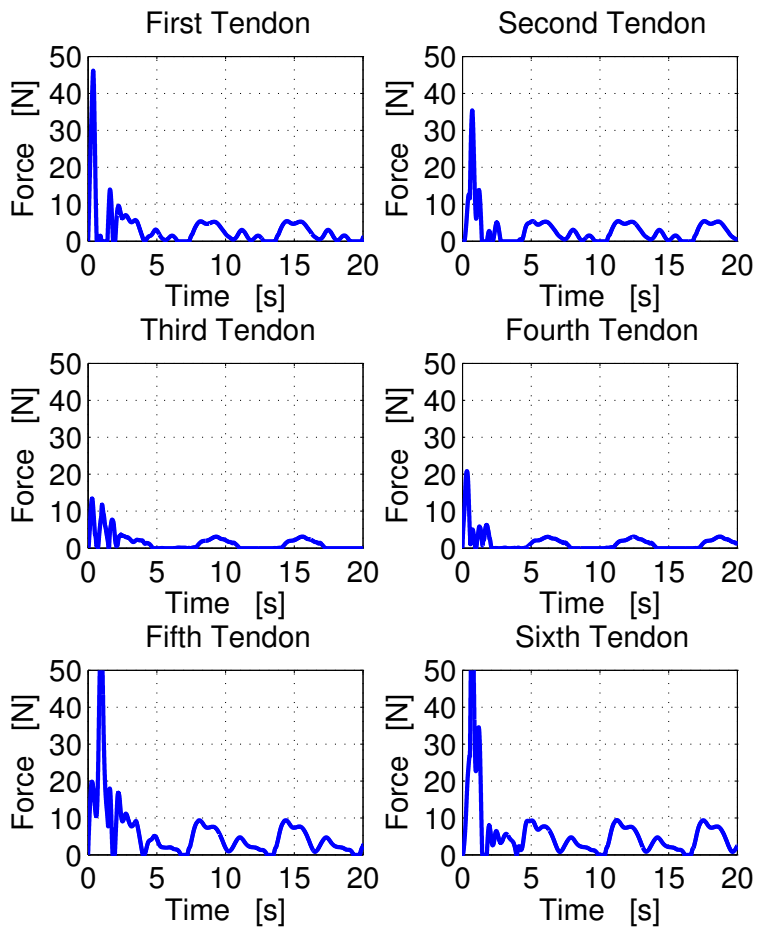

Fig. 4. Tendon tensile Forces

[9] G. Palli, "Model and control of tendon actuated robots," Ph.D. dissertation, Ph.D. dissertation, DEIS, University of Bologna, Bologna, Italy, 2007.

[10] J. J. Lee, "Tendon-driven manipulators: Analysis, synthesis and control," Ph.D. dissertation, Ph.D. Dissertation, Ph.D. 91-2 University of Maryland, College Park, MD, USA, 1991.

[11] T. Nakayama and H. Fujimoto, "Trajectory tracking control of tendon driven robots using delayed force feedback," in Proc. of Int. Conf. on Mechatronics, Kumamoto, Japan, 2007.

[12] S. K. K. Haiya and J. Hirai, "Control of tendon-driven robotic mechanism by nonlinear springs and hysteresis characteristics," in Proc. of IEEE Int. Conf. on Mechatronics, Malaga, Spain, 2009.

[13] — - "Tension control of tendon mechanisms by compensation of nonlinear spring characteristic equation error," in IEEE Workshop on Advanced Motion Control, Nagaoka, Japan, 2010, pp. 42-47.

[14] B. Okur, E. Zergeroglu, E. Tatlicioglu, and O. Aksoy, "Nonlinear adaptive partial state feedback trajectory tracking control of tendon driven robot manipulators," in Control Applications (CCA), 2014 IEEE Conference on, Oct 2014, pp. 228-233.

[15] M. Chalon and B. d'Andrea Novel, "Backstepping experimentally applied to an antagonistically driven finger with flexible tendons," in 19th IFAC World Congress, Aug 2014, pp. 217-223.

[16] C. T. A. F. L. Lewis and D. M. Dawson, Control of Robot Manipulators. New York, NY, USA: Macmillan Publishing Co., 1993.

[17] P. Tomei, "Adaptive pd controller for robot manipulators," IEEE Tr. on Robotics and Automation, vol. 7, no. 4, pp. 565-570, 1991.

[18] S. Nicosia and P. Tomei, "Robot control by using only position measurements," IEEE Tr. on Automatic Control, vol. 35, no. 9, pp. 1058-1061, 1990.

[19] T. Burg, D. Dawson, and P. Vedagarbha, "A redesigned dcal controller without velocity measurements: theory and demonstration," Robotica, vol. 15, pp. 337-346, 51997. 